\title{
Archilla Segade, H. (2019). Aportaciones en torno a la música y la educación musical. Cómo generar el cambio a través de la reflexión docente. Cáceres: Universidad de Extremadura. Servicio Editorial
}

El libro que aquí se reseña es fruto de un trabajo colaborativo entre docentes y conforma una obra coral que representa diferentes voces preocupadas por intentar mejorar la educación musical, por transformar las aulas y por el buen hacer. Este planteamiento compartido, esencial en la obra, es captado desde la misma presentación en la que el coordinador, Archilla, manifiesta explícitamente su ilusión por haberse podido materializar esta publicación "imaginada durante varios años". Con un marcado carácter didáctico, el texto completo ofrece una amplia paleta de experiencias musicales, en muchos casos interdisciplinares, realizadas en Educación Infantil, Primaria, Secundaria y, también, en el ámbito de la formación de profesorado. A lo largo de diez capítulos, los autores del libro tienen en común una apuesta por llevar a la práctica metodologías innovadoras que amplían las posibilidades expresivas y creadoras, lo que refleja un pensamiento docente reflexivo y un cuestionamiento educativo sobre lo establecido.

El primer capítulo aborda las canciones infantiles a través de una metodología que valora la tradición musical y tiene en cuenta, además, los grandes beneficios de la música desde las primeras edades aportando enfoques teóricos desde los ámbitos cognitivos y socioemocionales en el desarrollo infantil. Sus autores, Izaola y Correa, proponen el proyecto Musiquino, diseñado y adecuado al currículo de esta etapa. A través de cuadernillos de trabajo para el alumnado y una guía para el profesorado, este material didáctico ofrece una amplia variedad de actividades de alfombra, de movimiento y de mesa, a partir del repertorio de canciones populares. En el capítulo, se describe este material con numerosos ejemplos gráficos y organizado desde los tres años hasta los doce.

Musiversos es otro recurso educativo dirigido tanto a la etapa de Infantil como de Primaria y que surge desde un enfoque interdisciplinar entre la música y la poesía, cuyas conexiones ya desde la antigüedad y a lo largo de la historia de la música son apuntadas por la autora a lo largo de este segundo capítulo con numerosas referencias. Alcántara comparte también algunos libros de poemas propios como La Escaletra Fantástica, El Arcoíris de Colores, El Barquito Letrero y Glucolandia. La autora describe cada uno de ellos dejando un testimonio propio que trasluce su enfoque educativo globalizador y multiartístico y capaz de incorporar a las aulas los problemas actuales de manera inclusiva a través de proyectos donde la poesía es "un espacio común entre el texto, la palabra y la música". El texto recoge fotografías sobre las puestas en escena en los distintos lugares donde fueron realizados estos proyectos, así como ejemplos de poemas, cuentos, ritmos, canciones, dramatizaciones y toda suerte de actividades que a lo largo del capítulo los lectores podrán conocer. 


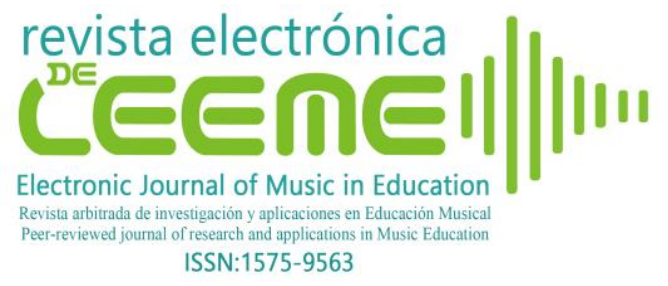

SECCIÓN DIFUSIÓN DE PUBLICACIONES: María Elena Riaño Archilla Segade, H. (2019). Aportaciones en torno a la música y la educación musical. Cómo generar el cambio a través de la reflexión docente. Cáceres: Universidad de Extremadura. Servicio Editorial (Revista Electrónica de LEEME) Número 47, pp. 98-102 https://ojs.uv.es/index.php/LEEME/index DOI: 10.7203/LEEME.47.19134

El emprendimiento educativo como reto para la escuela del siglo XXI es el punto de partida del autor de este tercer capítulo. Archilla pone el foco en una cultura que persigue el desarrollo del talento y la adquisición de competencias para que el alumnado sea capaz de resolver eficazmente las tareas. Para el autor, la figura docente es pieza clave en la consecución de este reto y, en el capítulo, se reflexiona sobre las metodologías de trabajo activas, participativas y que tengan en cuenta situaciones reales. En concreto, apuesta por el aprendizaje experiencial basado en el ciclo de Kolb. Desde esta perspectiva, Archilla comparte algunos proyectos desarrollados en las aulas de secundaria y relacionados con la música en el cine y en el audiovisual, en las que persigue desarrollar la capacidad emprendedora de los estudiantes. Se trata de experiencias creativas y colaborativas en las que se busca nuevas formas de motivar al alumnado a partir de sus propios intereses y del uso de las herramientas tecnológicas a su alcance. El uso de la música de cine en el proyecto Música y Óscars o de la música sinfónica como hilo conductor en el proyecto A que te lanzo la Batuta son excusas perfectas que el autor propone en sus proyectos musicales y multidisciplinares. Son experiencias de aula que tienen muy en cuenta las metodologías de trabajo y, también, las herramientas evaluativas según los estándares de aprendizaje, todo ello al servicio del logro de objetivos relacionados con el desarrollo de las competencias clave en sus estudiantes.

In Vino Veritas es el guiño que el autor del cuarto capítulo realiza al universo vinícola. Atance, desde una escritura reflexiva y poética, escribe un texto que ha de ser más que leído, degustado, al igual que se disfruta del aroma, el color o el sabor de un buen vino. A través de esta metáfora viticultora utilizada para hablar de educación, el autor establece una analogía entre el vino y los profesores. A lo largo del texto, se hace referencia a un imaginario sobre el que reflexionar: los caminos para la renovación, los tiempos de clase, los ideales, las experiencias personales, la calidad de la enseñanza, las problemáticas actuales o la formación. Atance asemeja en su narración los factores necesarios en la elaboración de un vino excelente (sol, tierra, lluvia e instalaciones bodegueras) con la excelencia del profesorado. En este proceso, el autor hace alusión a los tipos de vinos resultantes tras los años de necesarios para la adecuada crianza y lo compara con los años de experiencia del profesorado en las aulas. Bajo este relato sensorial subyace el verdadero propósito de este texto, que no es otro que el de lograr una enseñanza de calidad para el futuro de nuestras escuelas.

La enseñanza instrumental es planteada en el quinto capítulo mediante la incorporación de la guitarra en la educación musical. Díaz y Guerra defienden la búsqueda de un nuevo paradigma que integre elementos más allá de la melodía o la interpretación de un instrumento. La armonía, la incorporación de la voz, el uso de un repertorio diverso en estilos y motivante para el alumnado o el disfrute, tanto en la interpretación como en la creación, son algunas de las bases que motivan esta experiencia cooperativa e inclusiva realizada en Educación Secundaria. Se presenta en este capítulo la experiencia desarrollada fundamentada teóricamente y donde se exponen los objetivos, metodología, actividades, evaluación y los resultados obtenidos. Todo ello viene a reforzar una forma de trabajo en esta etapa educativa avalada durante una década 


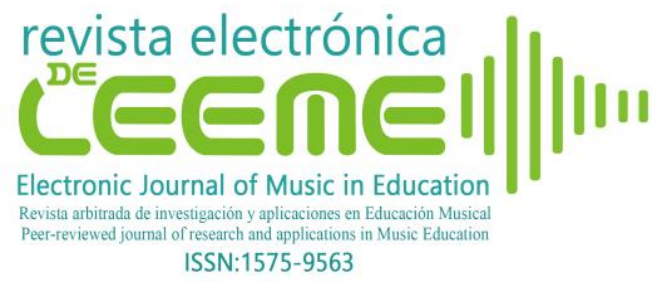

SECCIÓN DIFUSIÓN DE PUBLICACIONES: María Elena Riaño Archilla Segade, H. (2019). Aportaciones en torno a la música y la educación musical. Cómo generar el cambio a través de la reflexión docente. Cáceres: Universidad de Extremadura. Servicio Editorial (Revista Electrónica de LEEME) Número 47, pp. 98-102 https://ojs.uv.es/index.php/LEEME/index DOI: 10.7203/LEEME.47.19134

por los autores y que no solo avanza en el aprendizaje musical, sino que desarrolla aspectos extramusicales como la sociabilización, la cooperación, la interdisciplinariedad y la igualdad.

El sexto capítulo narra el encuentro de un grupo de docentes de música que comparten sus intereses en torno al trabajo creativo en las aulas. Desde la propia reflexión de su experiencia profesional, Murillo plantea rutas facilitadoras de la creatividad, abiertas y colaborativas, situando a la acción como plano central. El autor defiende la ruptura con la educación musical convencional y domesticada en aras de la búsqueda de la libertad expresiva para llegar al verdadero aprendizaje, donde lo emocional, lo cognitivo y lo social forman parte de un proceso abierto y experimental. Escuchar, confiar, asumir riesgos, replantear los tiempos o cambiar rutinas son algunas de estas rutas que preparan al lector para degustar tres propuestas creativas que fueron compartidas en el encuentro: PAMKATA, una experiencia desde el cuerpo cuyo detonante de acción fueron las sílabas rítmicas que conforman la palabra; Trazo en Corto, un juego de tarjetas con grafías abstractas que constituyeron un recurso de posibilidades interpretativas de gran potencial creativo; y Trazo en Largo, donde se introdujo la tecnología a través de los dispositivos móviles de un modo performativo que reforzó la creación sonora, el pensamiento creativo y el diálogo entre los docentes participantes en el encuentro.

García Gontán expone de manera clara y precisa en el séptimo capítulo los fundamentos teóricos de Soundpainting, un lenguaje gestual para la improvisación/creación estructurada en tiempo real, así como sus antecedentes, su evolución y los beneficios que otorga para el desarrollo de la capacidad creativa. Con un carácter didáctico, el autor incide en algunos aspectos clave a tener en cuenta en el procedimiento de trabajo con Soundpainting y que resultan esenciales tanto para el conductor (Soundpainter) como para los participantes. En el texto, se muestran algunas fotografías que ayudan a una mejor comprensión de la sintaxis de los gestos y otros signos. Posteriormente, el autor presenta tres experiencias didácticas de Soundpainting llevadas a cabo en contextos educativos desfavorecidos que promueven la inclusión e integración social. Estas experiencias constituyen prácticas reales que dan buena cuenta de las posibilidades integradoras, cognitivas, emocionales, performativas y musicales que ofrece el Soundpainting.

En el octavo capítulo, se presenta uno de los instrumentos más característicos de la tradición musical de nuestro país, la guitarra, como un recurso para las aulas. Horrillo cuestiona el escaso sentido de las prácticas musicales llevadas a cabo en las aulas cuando estas no parten de los intereses de los niños y jóvenes. Basándose en la motivación como enfoque de partida, el autor presenta una metodología propia y genuina que apuesta por repertorios musicales actuales y estilos como el pop y el rock. De manera práctica y sostenible, muestra la construcción de una guitarra escolar, concretamente del mástil, detallando tanto los materiales necesarios como el procedimiento a seguir. Se trata de un prototipo que permite al alumnado de $5^{\circ}$ de Primaria adquirir los fundamentos técnicos de la guitarra y los elementos propios del lenguaje musical, que sentará las bases para una futura interpretación del instrumento real. Horrillo realiza también, paso a paso, una descripción metodológica e incluye ejemplos gráficos de posiciones de acordes, tablaturas o ritmos. Finaliza el autor el texto abordando la evaluación que tiene en

@ María Elena Riaño. The content of this article is the sole responsibility of the authors. The Revista Electrónica de LEEME and Universitat de València are not liable for any legal actions that may arise involving the article's content. Revista Electrónica de LEEME - Lista Electrónica Europea de Música en la Educación-. http://ojs.uv.es/index/php/LEEME/index ISSN: 1575-9563. Editores: Universidad de Valencia y Jesús Tejada. Visibilidad de esta revista: SCOPUS, Emerging Sources Citation Index (Clarivate), EBSCO, CINDOC (CSIC), Citefactor, COPAC, Dialnet, DICE (CSIC), DOAJ, e-revistas (CSIC), EBSCO Premier, ERIH+, Gale Cengage Learning, INRECS, IRESIE, LATINDEX, MIAR, OCLC Worldcat, RESH, REDIB, RILM Core Journals, SUDOC, ULRICHS. Esta revista es de acceso libre mediante licencia Creative
Commons 4.0 CC by. Política de archivado: etiqueta verde SHERPA-ROMEO. 


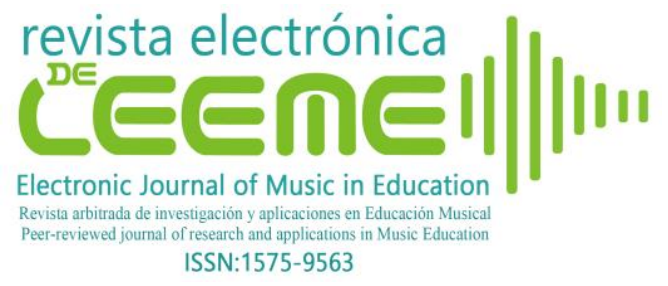

SECCIÓN DIFUSIÓN DE PUBLICACIONES: María Elena Riaño Archilla Segade, H. (2019). Aportaciones en torno a la música y la educación musical. Cómo generar el cambio a través de la reflexión docente. Cáceres: Universidad de Extremadura. Servicio Editorial (Revista Electrónica de LEEME) Número 47, pp. 98-102 https://ojs.uv.es/index.php/LEEME/index DOI: 10.7203/LEEME.47.19134

cuenta los estándares de aprendizaje del currículo y en la que incluye una rúbrica. La metodología presentada supone una forma de trabajar repertorios más amplios que apuesta por las agrupaciones instrumentales y las experiencias compartidas.

En el noveno capítulo, Díaz y Guerra ponen en valor una obra publicada por estos mismos autores anteriormente y titulada: La Música en Extremadura. Teoría y Práctica. Lo hacen partiendo de una profunda reflexión en torno a la importancia de que niños y jóvenes conozcan y tomen conciencia del enorme patrimonio musical heredado de individuos y grupos humanos a lo largo de diferentes épocas y lugares. Los autores brindan información sobre una vasta cantidad de músicos extremeños de reconocido prestigio y trayectoria profesional, lo que explica la escritura de una obra que recoge pasado y presente de la música en Extremadura. En este capítulo, Díaz y Guerra reseñan el libro, el cual contiene aspectos etnomusicológicos y didácticos. Así, las sugerencias metodológicas y las propuestas prácticas para el trabajo de la música en las aulas engloban actividades relacionadas con las canciones, los bailes y danzas, los instrumentos o los géneros. En la última sección, se hace referencia al sugerente material alojado en el repositorio Educarex con contenidos digitales materializados en forma de "objetos de aprendizaje", fundamentalmente auditivos, de gran utilidad para las etapas de Primaria y Secundaria.

El décimo y último capítulo está dedicado a la creación de un coro infantil en las etapas de Infantil y Primaria. Bajo la consideración del autor sobre la importancia de la práctica vocal como uno de los elementos principales de la experiencia del infante con la música y desde un enfoque lúdico, De los Reyes Cabrera expone y argumenta los elementos esenciales para crear un coro. El autor sugiere la idoneidad de los espacios, justifica el uso o no de materiales como las sillas, los instrumentos de acompañamiento o los objetos para el calentamiento físico. También, reflexiona sobre otros aspectos vinculados a la puesta en escena y sobre los agentes que participan en la formación y gestión del coro, más allá del propio alumnado, cuyos criterios de selección depende de las metas marcadas. Con sumo detalle, De los Reyes Cabrera relata la pedagogía de ensayo y describe técnicas y actividades tanto en la preparación corporal como vocal. Son todas ellas herramientas de ayuda e inspiración para cualquier docente interesado en la creación de coros infantiles en las aulas.

Realmente esta obra constituye una excelente aportación al ámbito educativo-musical que revertirá de manera directa tanto en el alumnado como en el profesorado. En consonancia con la esencia de las voces de los distintos autores que han dejado su huella en este libro, la escuela del siglo XXI requiere la construcción de comunidades que piensen juntas, pues la creatividad colectiva llega más lejos. En estas páginas, se ilustra perfectamente este pensamiento común que viene a arrojar luz en esta sociedad individualista donde se hacen necesarias propuestas colaborativas que fomenten espacios para la comunicación, la generosidad o el intercambio, entre otros valores. Todo un reto docente que hace necesario que el profesor se configure desde un fuerte compromiso profesional como un potente articulador para el cambio (Fuentealba y Imbarack, 2014). Y es desde una Educación Musical que cuestione lo establecido, que persiga este cambio, que amplíe la mirada y la escucha y que apueste por la

@María Elena Riaño. The content of this article is the sole responsibility of the authors. The Revista Electrónica de LEEME and Universitat de València are not liable for any legal actions that may arise involving the article's content. Revista Electrónica de LEEME - Lista Electrónica Europea de Música en la Educación-. http://ojs.uv.es/index/php/LEEME/index ISSN: 1575-9563. Editores: Universidad de Valencia y Jesús Tejada. Visibilidad de esta revista: SCOPUS, Emerging Sources Citation Index (Clarivate), EBSCO, CINDOC (CSIC), Citefactor, COPAC, Dialnet, DICE (CSIC), DOAJ, e-revistas (CSIC), EBSCO Premier, ERIH+, Gale Cengage Learning, IN-
RECS, IRESIE, LATINDEX, MIAR, OCLC Worldcat, RESH, REDIB, RILM Core Journals, SUDOC, ULRICHS. Esta revista es de acceso libre mediante licencia Creative Commons 4.0 CC by. Política de archivado: etiqueta verde SHERPA-ROMEO. 


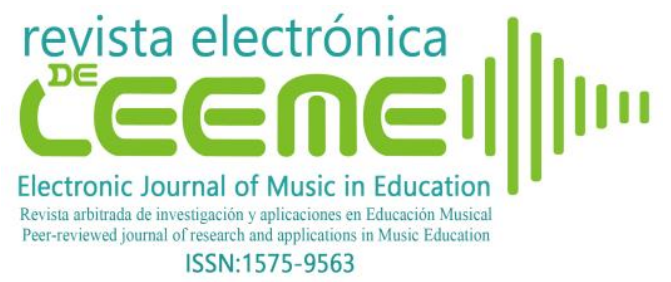

SECCIÓN DIFUSIÓN DE PUBLICACIONES: María Elena Riaño Archilla Segade, H. (2019). Aportaciones en torno a la música y la educación musical. Cómo generar el cambio a través de la reflexión docente. Cáceres: Universidad de Extremadura. Servicio Editorial (Revista Electronica de LEEME) Número 47, pp. 98-102 $\frac{\text { https://ojs.uv.es/10. }}{\text { DOI: 10.7203/LEEME.47.19134 }}$

creatividad, la colaboración y la innovación, cuando este cambio puede ser posible. Pues, en definitiva, la música es una de las formas más universales de expresión y comunicación del ser humano (Mehr et al., 2019) y la educación musical ayuda al desarrollo cognitivo, social y emocional de niños y jóvenes, contribuyendo positivamente a su salud y bienestar (Welch, Biasutti, MacRitchie, McPherson y Himonides, 2020).

\section{Referencias}

Fuentealba, R. y Imbarack, P. (2014). Compromiso docente, una interpelación al sentido de la profesionalidad en tiempos de cambio. Estudios pedagógicos (Valdivia), 40(ESPECIAL), 257273. doi: $10.4067 /$ S0718-07052014000200015

Mehr, S.A., Singh, M., Knox, D., Ketter, D.M., Pickens-Jones, D., Atwood, S., et al. (2019). Universality and diversity in human song. Science, 366(6468), 1-17. doi: 10.1126/science.aax0868

Welch, G.F., Biasutti, M., MacRitchie, J., McPherson, G.E. y Himonides, E. (2020). The Impact of Music on Human Development and Well-Being. Frontiers in Psychology, 11, 1246. doi: 10.3389/fpsyg.2020.01246

María Elena Riaño Galán

Facultad de Educación. Universidad de Cantabria

elena.riano@unican.es

@ María Elena Riaño. The content of this article is the sole responsibility of the authors. The Revista Electrónica de LEEME and Universitat de València are not liable for any legal actions that may arise involving the article's content. Revista Electrónica de LEEME - Lista Electrónica Europea de Música en la Educación-. http://ojs.uv.es/index/php/LEEME/index ISSN: 1575-9563. Editores: Universidad de Valencia y Jesús Tejada. Visibilidad de esta revista: SCOPUS, Emerging Sources Citation Index (Clarivate), EBSCO, CINDOC (CSIC), Citefactor, COPAC, Dialnet, DICE (CSIC), DOAJ, e-revistas (CSIC), EBSCO Premier, ERIH+, Gale Cengage Learning, INRECS, IRESIE, LATINDEX, MIAR, OCLC Worldcat, RESH, REDIB, RILM Core Journals, SUDOC, ULRICHS. Esta revista es de acceso libre mediante licencia Creative Commons 4.0 CC by. Política de archivado: etiqueta verde SHERPA-ROMEO. 